\title{
Improving healthcare performance by focusing on individual productivity of healthcare provider and system thinking: a strategy proposal
}

\author{
Choni Wangmo ${ }^{1}$
}

${ }^{1}$ Punakha District Hospital, Punakha, Bhutan

\begin{abstract}
Bhutan's health system, despite its commendable achievements in past few decades, continues to face shortage of skilled healthcare professionals and quality issues in service delivery. While recruiting more professionals, advocating for population health, better patient experience and safety, healthcare provider wellness has been overlooked. As the recent pay revision receives warm welcome from the health fraternity, a sense of greater professionalism and responsibility will be expected. Yet, aforementioned challenges still remain. Life and aspirations of professionals, brought up and trained in society that provides free healthcare and education, will become more convoluted. Mentorship program for healthcare providers at the beginning of their career and institutionalizing enabling environment for their personal and professional development need to be looked into. Decisions to pursue specialization should be a result of genuine interest and not a presumed exit hallway from professional frustrations, nor a shortcut to hefty pay. A promising and congruous career pathway can improve job satisfaction among health care providers. Endeavors to improve healthcare service delivery are present at every level but lack of coordination hampers favorable outcome. Variation in healthcare is major cause of adverse healthcare outcome and it is also known that every system is perfectly designed to achieve the results it gets. After conceptualizing few desired outcomes, a strategy framework that leads to health system improvement through improved individual productivity and systemic reforms enhancing intra- and inter sectoral coordination, while using evidence-based practice and existing resources, is proposed.
\end{abstract}

Keywords: Bhutan; Health provider wellness; Health system improvement; Quality improvement; System thinking

\section{Setting the context: healthcare system and health workers}

Bhutan started its planned development process in 1961 with the beginning of the $1^{\text {st }}$ Five Year Plan. Life expectancy in Bhutan has soared from 34.53 in 1960 to 71.13 years in $2017^{1}$. Maternal mortality and infant mortality have plummeted from 945(1990) \& 185.5 (1969) per 100000 live births to $121 \& 26$ in $2017^{2-4}$. Also, with the beginning of the Five-Year Plan, human resource requirements were clearly outlined and accordingly students were selected for training in India. Today majority of health workers graduate from the Faculty of Nursing and Public Health under the Khesar Gyalpo University of Medical Sciences of Bhutan (KGUMSB) but few professionals, including physicians still undergo their medical training outside Bhutan ${ }^{6}$.

Skilled health professional's density remains low in Bhutan (18.9 per 10000 population). It is below regional and global average of 25.7 and 52.8 per 10000 population respectively? While tackling with shortage of human and financial resources,

\section{Corresponding author:}

Choni Wangmo

choniwangmo@hotmail.com
Bhutanese health workers face additional challenge of keeping up with medical standards and innovations in other countries manifested in demand for high standard of care by patients.

Unfortunately, in recent times an increasing number of health workers are leaving the system, leading to further lowering of already small number. 24 doctors left practice between 2011 to 2015 and 15 between 2016 to 2018 (source: personal communication). This loss is urgent given that some specialties have only one physician trained in the field. Royal Civil Service Commission (RCSC) of Bhutan came up with Doctor's Career Path Reform to address this issue ${ }^{8}$. In spite of this, physicians (highly trained/experienced doctors and fresh graduates) are leaving the system. This has an impact on workload and quality of care delivered. For example, in 2015 a snake bite victim in Nanglam died because antivenom serum was not administered ${ }^{9}$. In 201811 neonates succumbed to hospital acquired infection in Jigme Dorji Wangchuck National Referral Hospital (JDWNRH) ${ }^{10}$. In April 2018, a patient with blunt trauma to abdomen died due to post-operative complications ${ }^{11}$. These cases caused quite a stir in the mainstream media and in some instances resulted in punitive action against the healthcare providers.

According to the 2017 National Healthcare Provider Job 
Satisfaction survey only $62 \%$ of the doctors were satisfied with their job, least amongst all categories of healthcare providers in the country ${ }^{12}$. This finding is concerning because physicians and their decisions have a huge impact on individual patient and overall health system outcome/expenditure ${ }^{13}$. Approximately $70 \%$ of healthcare costs are directly or indirectly controlled by physicians' decisions ${ }^{14}$. Another study found that recognition, aside from pay were considered important motivating factors for the Bhutanese physician ${ }^{15}$. In fact, until the recent pay revision begins to influence the decisions of future professionals, one can still say that physicians in Bhutan choose the profession for nonfinancial reasons. Majority of the physicians undergo medical training through merit-based government scholarship.

\section{Now, is the right time}

Medical history of Bhutan is at critical juncture today. While the government has rewarded the hard work of health workers with blanket pay rise, the health system needs to be redesigned to strengthen accountability and professionalism at every level. It needs to acknowledge the changes in needs and expectations of both patients/communities and the frontline workers. Professionals need to be encouraged to excel and enabled to explore ways to contribute better, rather than being coerced to follow regulations that were made when awareness on possibilities and resources to support were at its minimal.

"US Healthcare has focused on brilliant-the unusual diagnosis, the brilliant procedures, the latest medications... We have underemphasized the "routine", operational aspects of care, that arguably benefit more people" - William A Bornstein, MD. PhD.

Bhutan is on the other side of the spectrum. Our healthcare has been focused too much on the routine care for so long that now there is an emergent need of bold policy change nurturing brilliance and innovation to have any further improvement.

\section{Health workers'Early Career (especially physician)}

Fresh MBBS graduates after few weeks of orientation are sent to their first workplace, often on their own; and at times expected to manage healthcare center. These physicians experience numerous challenges and eventually, overs years most learn to navigate the highly complex healthcare system but, at a cost (can be anything from burnout to adverse patient outcome).

Bhutan's healthcare system should be designed to enable new recruits to learn from and be guided by leaders in the field. A mentorship program can alleviate the work-related stress and anxiety, increase job satisfaction, reduce turnover and promote personal and professional well-being ${ }^{17}$. Evidence suggests many areas of healthcare management - including staff recruitment and retention, education and training, quality improvement, patient safety, and risk management — can benefit from carefully planned, leadership-supported mentoring programs. By supporting internal learning experiences and relationships, organizations can foster improved performance and morale, nurture future leaders, and prepare to meet the many challenges and changes that will present in the years ahead ${ }^{18}$.

\section{Career Path}

Specialists are significantly more satisfied with their job than those with MBBS degree only ${ }^{15}$. Opportunity to pursue Residency has become easier in last six years with the beginning of the FoPGM in KGUMSB. However, timing and regulations related to opportunities outside KGUMSB has not been clear. Few have been able pursue specialization right after undergraduate studies. Some never get to pursue the specialization in the field of their interest. Some get selected for government scholarship but fail to get admission to master's program. Some get admission to a master's program but do not get financial support. Some manage to find admission and financial support from elsewhere but do not get approval to leave for study. Additionally, even after years of administrative work, a physician is not accepted as a

Table 1. A summary of current situation

\begin{tabular}{|c|c|c|}
\hline Good & Bad & Ugly \\
\hline 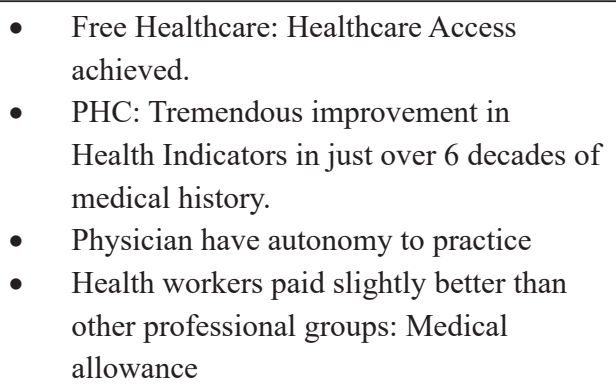 & $\begin{array}{ll}\text { - } & \text { Quality of care not assessed } \\
\text { - } & \text { Patient expectation not met } \\
\text { - } & \text { No incentive to drive innovation } \\
\text { - } & \text { High workload } \\
\text { - } & \text { No system thinking } \\
\text { - } & \text { heatients and physicians not mindful of } \\
\text { Limited opportunity to pursue interest }\end{array}$ & $\begin{array}{ll}\text { - } & \text { Brain drain, while there is shortage of } \\
\text { - } & \text { Phofessionals } \\
\text { - } & \text { Adverse healthcare outcomes } \\
\text { - } & \text { Punitive action against medical errors }\end{array}$ \\
\hline
\end{tabular}

\section{What needs to change? Why? and the desired outcome}

1 Excerpt from Lecture on Health System Improvement for Physician

Performance course Spring 2019, Department of Health Policy and

Management, Rollins School of Public Health, Emory University. 
"manager" to be able to proceed in executive career pathway of civil service. At a glance solution seems as simple as equal opportunity to study further before joining civil service, equal opportunity to pursue one's field of interest and equal opportunity to choose a career path suitable to one's aptitude. But, for some reason it hasn't worked out that way. Perhaps, a separate agency is required to look into this several decades old issue. Our system should recognize the importance of every specialty, including primary care and hospital administration. Healthcare provider wellness and professional development opportunities need to be looked into. Leadership capable of envisioning and enabling such systemic improvement is needed. Interdisciplinary collaboration within the health system should be enabled before embarking into intersectoral ventures. So far one can say that health related intersectoral and interdisciplinary collaboration has been unidirectional.

\section{Unstandardized practice}

There is huge variation in healthcare ${ }^{19}$. In Bhutan, this variation is worsened by the fact that physicians are trained in universities across different countries. Few Programs in $\mathrm{MOH}$ attempt to standardize treatment through trainings and distribution of guidelines. However, with no mechanism to track, implementation is left completely up to the discretion of health workers. Further, in spite of being public hospitals run by the same government, each health center in the country has its own way of working. There is a confusion on roles and responsibilities of physician at different levels. This unstandardized patientcare and organizational management not just lead to inefficiency but increase risk of adverse health outcomes. Standardization of care \& management, with periodic monitoring and evaluation would address most of the common issues in the health system. Clinical audits with timely feedbacks could prevent unforeseen grave adverse medical outcome. All of these would need to be done at health center level (hospitals and BHUs) and managerial roles assumed by physicians should be formalized to ensure long-term planning.

\section{Intellectual Stasis and Emotional Isolation:}

Most of the physicians are practicing what they remember from their medical training. Learning and following guidelines are pretty much left up to individual physician's convenience. When errors occur, which happens very often in healthcare ${ }^{19}$, they change their strategy as deemed necessary by themselves. There are no external measures to monitor or guide them. Physicians are emotionally devastated by serious mistakes that harm or kill patients ${ }^{20-22}$. Almost every physician who cares for patients has had that experience, usually more than once. The emotional impact is often profound, typically a mixture of fear, guilt, anger, embarrassment, and humiliation. Physicians are typically isolated by their emotional responses; seldom is there a process to evaluate the circumstances of a mistake and to provide support and emotional healing for the fallible physician ${ }^{21}$. From an emotional standpoint, they need the support and understanding of their colleagues and patients when they make mistakes. Yet, they are denied both insight and support by misguided concepts of infallibility and by fear: fear of embarrassment by colleagues, fear of patient reaction, and fear of litigation ${ }^{23}$.

This is a worrisome situation because physicians are the cornerstone of health delivery system. Health-care costs ultimately arise from the accumulation of individual decisions doctors make about services and treatments to write an order for. The most expensive piece of medical equipment, as the saying goes, is a doctor's pen ${ }^{24}$. Also, by nature of this discipline, a physician has to continue learning and being abreast of the latest guidelines. Health system should be redesigned into one that is emotionally supportive and conducive for physicians to participate in academically beneficial activities while providing quality care to the patients.

\section{Adding EMR to the eHealth Strategy}

National eHealth Policy of Bhutan states that "Digitized Health record and information system shall be instituted in all the health facilities for faster and effective health information generation to support decision making" 25 . Even if the existing system is perfectly integrated, the reliability will remain an issue because interactions at individual level are still not digitalized. ICT tools that would help a physician track the past history of patients and support clinical decision through reminders and best-practice guidelines for treatment have not been discussed explicitly in the current eHealth Strategy.

Introduction of Electronic Medical Record (EMR) could help address the quality issues in direct patient care. It will also improve communication, coordination, measurement and decision support.

In 2005, a study in US concluded that effective EMR implementation and networking could eventually save more than $\$ 81$ billion annually by improving healthcare efficiency and safety-and that HIT (Health Information Technology)-enabled prevention and management of chronic disease could eventually double those savings while increasing health and other social benefits ${ }^{27}$.Others say healthcare expenditure and physician burnout has increased as a result of EMR introduction. And that there have been few savings from the $\mathrm{EMR}^{28}$.

EMR could be a success story in Bhutan because firstly there will be just one system for the whole country. Secondly, Bhutan could wait for few years before incentivizing any activity related to EMR use. One important amendment to current eHealth strategy should be involvement of users in the early stage. A customized, reliable and efficient EMR system needs to be developed. ICT officers who work in this project should be closely involved with professionals in hospitals and understand the work process. We would not want to invest in a healthcare improvement tool that virtually locks out the users in long run. 
Develop a tradition that insists on the collection and evaluation of information to understand and describe the consequences of medical practices

Healthcare workers can do more to admit the existence of uncertainty, both to themselves and to their patients. While this will undoubtedly be unsettling, it is honest, and it opens the way for a more intensive search for ways to reduce uncertainty ${ }^{19}$. Individuals who want to promote policies regarding use of medical procedures could learn the necessary languages (statistics, probability theory, mathematics and economics and decision theory). Physicians should be encouraged to publish papers that synthesize existing information, estimate the outcomes of different policies, and present the rationales for different actions. Government can support more evaluation research to analyze medical practices. Patients can push the process by asking questions. The problems that exist today are not the fault of any individuals; the fault lies with the profession and society as a whole for not developing the traditions and methods needed to assess medical practices ${ }^{19}$. We certainly cannot blame a systematically chosen Grade 12 student for choosing the profession seven years later.

\section{Pay for performance}

Policy experts believe payment method reforms for healthcare providers is the most promising method of improving the quality of care and controlling costs ${ }^{29}$. Some say Pay for Performance (P4P) results in unsustainable post-introduction initial quality improvement acceleration, with possibility of unintended consequences, including reductions in the quality of some aspects of care not linked to incentives and in the continuity of care ${ }^{30}$. For Bhutan, $\mathrm{P} 4 \mathrm{P}$ is implementable especially because of small number of physicians. It could also be used to reward teams, and in that way bind physicians and other health workers together in seeking the same patient outcome objective. However, unfamiliarity to such a system, comfort of generalizing corrective action and possibly even lack of expertise has contributed to non-acceptance of an elementary proposal like Doctors Oncall Allowance by the recent $5^{\text {th }}$ pay Commission of Bhutan. The Oncall allowance could have been the first step towards P4P system.

Having said that, P4P at the individual level would slowly but surely happen as the country's human resource capacity develops. Meanwhile, performance evaluation of hospitals based on quality improvement measures can be started. This is more doable as the Quality Assurance \& Standardization Division (QASD) in $\mathrm{MOH}$ has already laid the framework for assessment. KPIs (Key Performance Indicators) in the Bhutan Health Standard Quality Assurance (BHSQA) guideline could be used to rank and reward the hospitals performing well. Systemic improvement in hospitals will definitely lead to improvement in overall health system.

\section{Healthcare expenditure and Systems approach}

Atul Gawande in his article "The Cost Conundrum" recommends cities wean away from untenably fragmented, quantity-driven systems of health care ${ }^{24}$. He says doctors and hospitals should be rewarded if they collaborate to increase prevention and improve quality of care, while discouraging overtreatment, undertreatment, and sheer profiteering. He says government could shift regulatory burdens, and even malpractice liability, from the individual to organization. He recommends in-depth research on what makes the best systems successful. Further he says a national institute for health-care delivery, that regularly assess the quality and the cost of care, review the strategies that produce good results, and make clear recommendations for local systems should be formed.

As mentioned earlier, health system in Bhutan is very different from that in US but we can definitely learn a lot from each other. The recommendation Gawande makes is pertinent to our health system as well. In fact, one can even say that we are on the right track. Unlike few decades earlier we have the insight and access to resources. Important foundations are already laid and some entities have already started to address issues. The challenge is to come up with a mechanism to coordinate.

\section{How do we get to our desired outcome?}

\section{Strategy Framework:A proposal}

Each of the desired outcomes proposed above lead to improved Health System Performance through improved individual productivity and improved coordination within healthcare system.

\begin{tabular}{|c|c|c|}
\hline Desired outcome & Focus & Goal \\
\hline $\begin{array}{ll}\text { - } & \text { Support during early } \\
\text { career } \\
\text { - } \\
\text { - } \\
\text { Clear career path } \\
\text { involvemic } \\
\text { - } \\
\text { - } \text { Better Job satisfaction } \\
\text { Hospital performance } \\
\text { evaluation } \\
\text { - Add EMR to eHealth } \\
\text { Strategy } \\
\text { - } \quad \text { Standardized care } \\
\text { - } \text { Better quality } \\
\text { healthcare } \\
\text { - System Thinking }\end{array}$ & $\begin{array}{l}\text { Improve } \\
\text { healthcare } \\
\text { quality } \\
\text { through } \\
\text { improved } \\
\text { coordination }\end{array}$ & $\begin{array}{l}\text { Improved } \\
\text { Health System } \\
\text { performance }\end{array}$ \\
\hline
\end{tabular}

\section{Figure 2. BCMP strategy framework}

The framework summarized in Figure 2 emphasizes the fact that healthcare provider performance is very much a part of health system. One cannot make any changes to healthcare provider performance without changing the health system and vice versa. Physician (a healthcare provider) performance is rightly said to be $85 \%$ system related ${ }^{31}$. 


\begin{tabular}{|c|c|c|c|}
\hline Objectives & Performance Improvement Approach & Measure & Agency responsible \\
\hline \multirow[b]{2}{*}{$\begin{array}{l}\text { 1.Improve } \\
\text { healthcare quality } \\
\text { through improved } \\
\text { coordination }\end{array}$} & $\begin{array}{l}\text { 1.Standardization of care with regular monitoring and } \\
\text { evaluation both at Hospital level and National level. }\end{array}$ & BHSQA-KPI* & $\begin{array}{l}\mathrm{QASD}^{\dagger} \text { and } \mathrm{DHS}^{\ddagger} \text { in } \\
\mathrm{MOH}, \mathrm{KGUMSB}\end{array}$ \\
\hline & $\begin{array}{cll}\begin{array}{l}\text { 2.Ranking } \\
\text { outcomes. }\end{array} & \text { and rewarding (incentivizing) quality } \\
& \text { - Individuals at hospital level } \\
& \text { - Hospitals at national level }\end{array}$ & Health outcomes & $\begin{array}{l}\text { DHS and } \mathrm{DH}^{\S} \text { in } \\
\mathrm{MOH}, \mathrm{RCSC}\end{array}$ \\
\hline \multirow{4}{*}{$\begin{array}{l}\text { 2.Improve } \\
\text { individual } \\
\text { productivity }\end{array}$} & 3.Include EMR in eHealth Strategy & Implementation of EMR & ICTD, MOH \\
\hline & 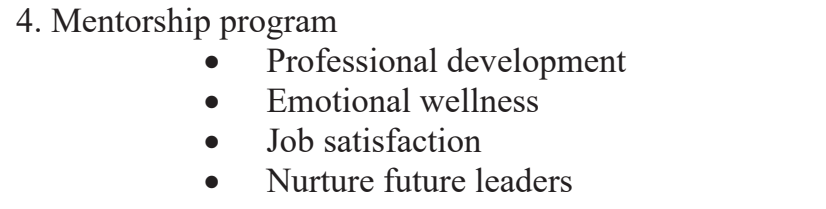 & $\begin{array}{l}\text { Health Worker Job } \\
\text { Satisfaction and Patient } \\
\text { Satisfaction scores }\end{array}$ & $\mathrm{MOH}, \mathrm{RCSC}$ \\
\hline & $\begin{array}{l}\text { 5.Streamline career path } \\
\bullet \quad \text { Equal opportunity to pursue } \\
\text { specialization field of interest including } \\
\text { MPH } \\
\bullet \begin{array}{l}\text { Job responsibility congruent with } \\
\text { reality }\end{array}\end{array}$ & Staff turnover & $\mathrm{MOE}, \mathrm{MOH}, \mathrm{RCSC}$ \\
\hline & $\begin{aligned} \text { 6. Professional group formation } \\
\bullet \quad \text { Promote research culture } \\
\bullet \quad \text { Develop a platform to share practice } \\
\text { success and experience } \\
\bullet \quad \begin{array}{l}\text { Improve healthcare by participating in } \\
\text { policy decision making, innovation }\end{array}\end{aligned}$ & $\begin{array}{l}\text { Research activity, } \\
\text { participation in policy } \\
\text { decision making, } \\
\text { academic learning, } \\
\text { sharing experiences } \\
\text { and success stories in } \\
\text { journals }\end{array}$ & $\begin{array}{l}\text { Health professionals } \\
\text { especially physicians }\end{array}$ \\
\hline
\end{tabular}

${ }^{*}$ BHSQA-KPI: Bhutan Health Standard Quality Assurance- Key Performance Indicators, ${ }^{\S}$ DH: District Hospital

$Q A S D$ : Quality Assurance \& Standardization Division

${ }^{\ddagger}$ DHS: District Health Service Program, ICTD: Information Communication and Technology Division, MOH: Ministry of Education

Figure 3. Aim of BCMP: Improve Health System Performance

To reach our goal we could form a separate independent professional agency/body, say for example an entity named Bhutan Center for Medical Professionalism (BCMP), comprised of experts from multiple health related disciplines. It would enable health professionals to attain their highest potential and ensure quality healthcare delivery through systemic reform. This extremely important and responsible institute should epitomize professionalism, high ethical standards, respect, support, competence and expertise in patient safety and healthcare provider wellness.

As proposed in Figure 3, the BCMP could coordinate the six major Health System improvement approach. Standardization of care with regular monitoring and evaluation will be one of the most challenging tasks given the complexity of public hospitals and unavailability of digital medical data at present. However, we can use KPI of BHSQA to start-of. With this performance-based hospital ranking and rewarding would be possible.
A Future Leaders Mentoring Programme was introduced by RCSC in 2017 as one of the programs under the Civil Service Well-being reform ${ }^{32}$. BCMP could propose for a similar program suitable for medical professionals. Given the nature of work, physicians could benefit immensely from a formal program like this.

BCMP will guide the physicians in forming a professional group. Forming a professional support group to ensure healthy discussions and active learning won't just boost the performance and morale of the physicians but germinate a possibility of Bhutanese physicians inventing and innovating in medical field. So far, work environment of physicians has not been favorable for academic growth. They do not have a formal platform to interact with fellow-physicians and share their experiences and success stories. There is neither a medium for sharing genuine concerns nor a nurturing and non-judgmental support mechanism for grievances. 


\section{Rationale for choosing this strategy}

This strategy was chosen because it holds a certain body responsible. It addresses various challenges faced by Bhutanese health professionals. A systemic and collaborative approach like this is required because in healthcare there is high interdependency within and across the system. BCMP will work independently but closely with the Ministry of Health, Royal Civil Service Commission and Bhutan Medical and Health Council (BMHC). Evidence based practice will be instrumental in changing the social norms. Having BCMP will give assurance to health professionals that there is an agency that looks after their welfare. Current BMHC rules and regulations does that for the patients.Mostly importantly this strategy is culturally acceptable. It's foundation on evidence-based practice and ultimate aim of improving healthcare is more acceptable to societal environment. It follows the "new Donabedian Equation."- Dr. William A. Bornstein:

\section{$\mathbf{S}+\mathbf{P}+\mathbf{C}=\mathbf{O}$ \\ Structure + Process + Culture $=$ Outcome}

Note: Healthcare is a team effort. Although throughout history of medicine physicians have led the profession, which still remains true in other countries, Bhutan has given equal importance to all the professionals honoring the contributions made by each category of healthcare providers. However, findings that suggest that physicians are not just the least satisfied employees amongst the healthcare providers ${ }^{12}$ but also much less happy than the general population $^{33}$ is something to worry about. These professionals, constituting only $10 \%$ of the health workforce ${ }^{3}$, are not just the leaders of patient treatment team but also often the managers of healthcare centers. They are overworked and drained from high expectations. Most importantly, much evidence suggests that the majority of the healthcare outcome and expenditure is dependent on them. Therefore, it is crucial to make a concerted effort to improve healthcare system with focus on physician performance.

\section{REFERENCES}

1. Online Archive of Life expectancy at birth, total (years) Bhutan [Internet]. Washington, DC: The World Bank. 1960[cited 2019 Oct 10]. [Full Text]

2. Online Archive of Maternal mortality in 1990-2015: Bhutan [Internet]. Maternal Mortality Estimation Inter-Agency Group, Bhutan. 1990- [cited 2019 Oct 10]. [Full Text]

3. Ministry of Health. Annual Health Bulletin, 2017. Thimphu: Ministry of Health, Royal government of Bhutan; 2018.

4. Online Archive of key demographic indicators Bhutan, 2018 [Internet]. UNICEF, Bhutan. 1990- [cited 2019 Oct 10]. [Full Text]
5. Ministry of Health RGoB. Overview [Internet]. Thimphu: Ministry of Health, Royal government of Bhutan; 2019 [cited 2019 Oct 15]. [Full Text]

6. Dorji T, Melgaard B. Medical history of Bhutan: a chronicle of health and disease from Bon times to today. $2^{\text {nd }}$ ed. Thimphu: Centre for Research Initiatives; Aug 17 2018. 303$30 \mathrm{p}$.

7. Online Archive of Health workers density and distribution: health workers density and distribution, 2018 [Internet]. WHO. [cited 2019 Oct 10]. [Full Text]

8. Amendment to sections of BCSR 2012 as per Doctor's career path reform, Bhutan Civil Service Regulations of 2012. Rep. No. RCSC/LS-63/2015/1447 (Nov.26, 2015). [Full Text]

9. Pokhrel N. Family accuses Nanglam doctor of negligence. Kuensel [Internet]. 2016 May 31;Sect.:1 (col.1).

10. Tshomo D. 24 babies infected in NICU- 15 survive, 9 dies:JDWNRH. Kuensel [Internet]. 2018 September 13; Sect.:1 (col.1).

11. Reporter S. Committee to investigate negligence in patient's death at JDWNRH. Kuensel [Internet]. 2018 May 18; Sect.:1 (col.1).

12. Tshomo D. $68 \%$ of healthcareproviders satisfied with their job. Kuensel [Internet]. 2017 September 19; Sect.:1 (col.1).

13. John M. Eisenberg MD. Doctor's decisions and the cost of medical care. Ann Arbor, Michigan: Health Administration Press Perspectives, 1986.

14. Volpp KG, Schwartz JS. Myths and realities surrounding health reform. Jama. 1994;271(17):1370-2 p.

15. Wangmo C, Palzang T, Zhang T, Quick R. A cross-sectional job satisfaction survey of Physicians in Bhutan to address the problem of retention. Bhutan Health Journal. Forthcoming 2019.

16. Saltman RB. Health Care Systems in Developed Countries. Harrison's Principles of INTERNAL MEDICINE. 1. 20 ed: Mc Graw Hill Education; 2018. p. 27-33.

17. Choudhury T. 7 Benefits of a structured workplace mentoring program 2018. [Full Text]

18. Schmidt B. Mentoring Programs: Essential for Sustaining a Culture of Safety: PSQG: Patient Safety \& Quality Healthcare; 2013. [Full Text]

19. Eddy DM. Variations in Physician practice: the role of uncertainty. Health Affairs. 1984;3(2):74-89.

20. Hilfiker D, M.D. Facing our mistakes. N. Engl. J. Med. 1984;310(2):118-22.

21. Christensen JF, Levinson W, Dunn PM. The heart of darkness. J. Gen. Intern. Med. 1992;7(4):424-31.

22. Wu AW, Folkman S, McPhee SJ, Lo B. Do House Officers learn from their mistakes? JAMA. 1991;265(16):2089-94. 
23. Leape LL. Error in medicine. JAMA. 1994;272(23):1851-7.

24. Gawande A. The cost conundrum: What a Texas toen can teach us about health care. The NEW YORKER. 2009 June 12009.

25. Ministry of Health. National Health Policy. Bhutan: 2011. $28 \mathrm{p}$.

26. Ministry of Health. National eHealth strategy and action plan. Bhutan: 2018 June. 45 p.

27. Can Electronic Medical Record Systems Transform Health Care? Potential Health Benefits, Savings, and Costs. Health Affairs. 2005;24(5):1103-17. [Full Text]

28. Reed Abelson JC. In Second look, Few Savings From Digital Health Records. The Neew York Times. January 10, 2013 January 102013.

29. Ginsburg PB. Rapidly evolving physician-payment policy more than the SGR. N. Engl. J. Med. 2011;364(2):172-6.
30. Campbell SM, Reeves D, Kontopantelis E, Sibbald B, Roland M. Effects of pay for performance on the quality of primary care in England. N. Engl. J. Med. 2009;361(4):36878. [Full Text | DOI]

31. Deming WE. Elementary principles of the statistical control of quality a series of lectures. $2^{\text {nd }}$ rev. ed. Tokyo: Nippon Kagaku Gijutsu Remmei; 1952.

32. Online Archive of Future Leaders Mentoring programme (FLMP) [Internet]. Royal Civil Service Commisssion, Bhutan. 2017- [cited 2019 Oct 10]. [Full Text]

33. Dem U, Swartz M, Mirecki I, Barak Y. Physicians' life satisfaction in Bhutan: a nationwide survey. Open Journal of Psychiatry. 2016;06:119-24. [Full Text | DOI] 